\title{
A secured tag for implementation of traceability in textile and clothing supply chain
}

\author{
Tarun Kumar Agrawal ${ }^{1,2,3,4} \cdot$ Ludovic Koehl $^{1,2} \cdot$ Christine Campagne $^{1,2}$ \\ Received: 25 March 2018 / Accepted: 22 August 2018 / Published online: 6 September 2018 \\ (C) The Author(s) 2018
}

\begin{abstract}
Textile and clothing industry is one of the oldest manufacturing industries and is a major contributor in the economic growth of developing countries. However, from past few decades, it has been criticised for its opaque, unsecured and untraceable nature of supply chain. Addressing these challenges, the paper proposes a system approach to introduce an item-centric secured traceability concept to monitor and control manufacturing processes and supply chain activities. In order to implement such secured traceability system, the paper describes the process for manufacturing, encoding and validating an innovative two-factor secured tag based on particle randomness that is printed on the surface of textile. Being micro-sized, the particles are easy to read and validate with pattern recognition. Further, as achieved through an uncontrolled manufacturing process, the randomness is unclonable to produce counterfeit tags. Furthermore, a sequence of experimental analyses has been conducted using various simulated scenarios to verify its applicability. A secured tag can be a low-cost and durable substitute for detachable, unsecured identifiers commercially available in the market.
\end{abstract}

Keywords Supply chain management $\cdot$ Manufacturing $\cdot$ Traceability $\cdot$ Textile and clothing $\cdot$ Secured tag

\section{Introduction}

Supply chain transparency and security have emerged as key concerns for various industries. With growing number of ethical issues and increasing counterfeits, it has become a necessity to make the supply chains more secure and transparent [1-3]. Textile and clothing (T\&C) supply chain is one such example. Complex supply chain structure with large geographical distance among actors has made the $\mathrm{T} \& \mathrm{C}$ sector one of the most opaque and vulnerable networks [4]. Although all T\&C supply chain partners are linked together as a network, yet, they do not have sufficient information

Tarun Kumar Agrawal

tarun-kumar.agrawal@ensait.fr; tarun_kumar.agrawal@hb.se; tarunagraw@gmail.com

1 ENSAIT, GEMTEX - Laboratoire de Génie et Matériaux Textiles, 59000 Lille, France

2 Université Lille Nord de France, 59000 Lille, France

3 The Swedish School of Textiles, University of Boras, 50190 Boras, Sweden

4 College of Textile and Clothing Engineering, Soochow University, Suzhou, China about their supply chain. Owing to this, suppliers and other involved actors use unethical means of production and distribution, which have resulted in several catastrophic events in the past. This has distressed the governments and drew their attention towards the sustainability issues in T\&C industries [5].

Security and counterfeits are other major concerns of the T\&C supply chain [6]. According to McMillen et al. [7], manufacturing industries including $\mathrm{T} \& \mathrm{C}$ have experienced a high level of security issues in past few years. In the European Union (EU), T\&C sector has lost more than EUR 26 billion of revenue in the years 2010-2011, resulting in approximately 500,000 direct and indirect jobs and EUR 17 billion sales loss in the related sectors [8]. Unlike other sectors, the problem of counterfeits in T\&C supply chain is not only limited to the final product. It is also a major concern in the intermediate products [6]. The fake products not only result in financial losses for the companies but also endanger the health and well-being of the end users [9].

Adoption of a secured traceability system has emerged as a possible solution to overcome the aforementioned problems $[10,11]$. Traceability is a key concept related to all types of products and supply chains [12]. It is an itemcentric approach for tracking and tracing a product [13]. It generally uses a unique identifier tag (such as RFIDs, QR 
codes and barcodes) assigned to each traceable unit. The tags track the products at various levels of the supply chain. However, they are often detached, removed or deactivated at the point of sale due to privacy concerns [14]. Moreover, most of the tags can easily be replicated and do not have any security mechanism for authentication. As a result, the T\&C market is vulnerable to unsafe, counterfeit products, and often, the internal operational information cannot be recalled which may expose the end users to serious security concerns. For instance, clothing is one of the most recalled products [15], albeit only a small fraction of items are taken back from market due to lack of traceability. In this direction, barcodes (such as linear barcodes and QR-codes) are generally used to track products in the supply chain. On one hand, the barcodes are relatively cheap to manufacture and implement [16], they do not create privacy concerns (unlike RFID) on the other hand [17]. Moreover, linear barcodes and QR codes are eco-friendly and degradable [16]. With selection of appropriate printing mechanism and ink, they can be made waterproof and durable. These characteristics make them appropriate for application in $\mathrm{T} \& \mathrm{C}$ product.

In the context of implementing secured traceability, the present paper introduces a two-factor product authentication and tracking system for T\&C supply chain. With the view of securing the existing QR code, a random particlebased secured tag as an additional security layer has been introduced. The unique and random feature of each secured tag is associated with the corresponding QR code. This combination acts as a key to unlock the traceability code, authenticates the product and traces its history. Unlike widely used detachable RFID and barcode tags, the proposed system is to be integrated onto the surface of the textile product. This makes the tag almost inseparable, preventing loss or replacement with counterfeits in the supply chain. Produced by conventional textile printing mechanism, the tags are anticipated to be of low-cost and easy to manufacture on a large scale. This can be one of the useful contributions to the $\mathrm{T} \& \mathrm{C}$ supply chain with low-cost product wherein a slightly higher investment in the traceability technology can increase the final product cost and reduce profit margin. Particle randomness generated during printing process with no control on the distribution acts as a unique feature for each product, which is hard to replicate. This randomness is read and encoded as a secured code to access the product database through the QR code and prevent it from cloning. To prove the uniqueness and evaluate its performance, a series of experiments have been conducted which simulate various mechanical agitations that a textile tag usually undergoes during its use phase. Although the proposed system is developed for T\&C supply chain, the methodology can be used for other similar supply chains.

\section{Literature review}

According to (ISO 9000:2015), 'Traceability is the ability to identify and trace the history, distribution, location, and application of products, parts, materials, and services. A traceability system records and follows the trail as products, parts, materials, and services come from suppliers and are processed and ultimately distributed as final products and services'. It has multiple benefits including transparency, enhanced quality perception, and better control of the material flow $[18,19]$. Traceability is a useful tool to overcome challenges in an omnichannel retailing and to reduce the risks in supply chain by identifying the upstream source and downstream recipients in real time. It further helps in decision making, product recall, recycling and remanufacturing. Its applications can also be explored for shared economy business models where traceability can act as an authenticating mechanism and information related to the history, origin, date of purchase, etc. could be shared at customer-to-customer level.

A significant research has been carried out in the past to develop various technologies to prevent counterfeiting and/or tracking and tracing the product in a supply chain [20-22]. There are authentication technologies that aim to identify counterfeits without tracing the product footprint in the supply chain. On the other hand, there are track and trace technologies which are used for locating the products within the supply chain. It relies on tagging each product or group of products with a unique identifier such as RFIDs and barcodes, and then these identifiers are used as a tracing element. However, due to lack of security mechanism, these identifiers are cloned or replicated and then used with counterfeit products [23]. As a result, track and trace systems cannot rely solely on traceability tags and requires an additional security mechanism for authentication and prevention of unauthorised reproduction/cloning [10]. Such system is often known as a 'secured traceability' system [11] and can be found in food, pharmaceutical, cosmetics and luxury product supply chain $[10,11,24]$. Table 1 provides an overview of the various technologies and their pros and cons with respect to product authentication, tracking and security.

In general, the security mechanisms in a secured traceability system are applied at two levels; first, at a product level, where authentication and traceability are ensured by identification and tracking of each product using unique featurebased secured traceability tags; second, at a business level, where information flow is controlled and secured by using mechanisms such as 'circle of trust' and 'block chain' [10].

Given the peculiarities of the T\&C supply chain, the traceability requirements are special and it cannot use offthe-shelf solutions in the market [40]. Durability, clonability and detachability of the traceability tags are some of the major issues in T\&C products [4]. A secured traceability tag for textiles should have security 


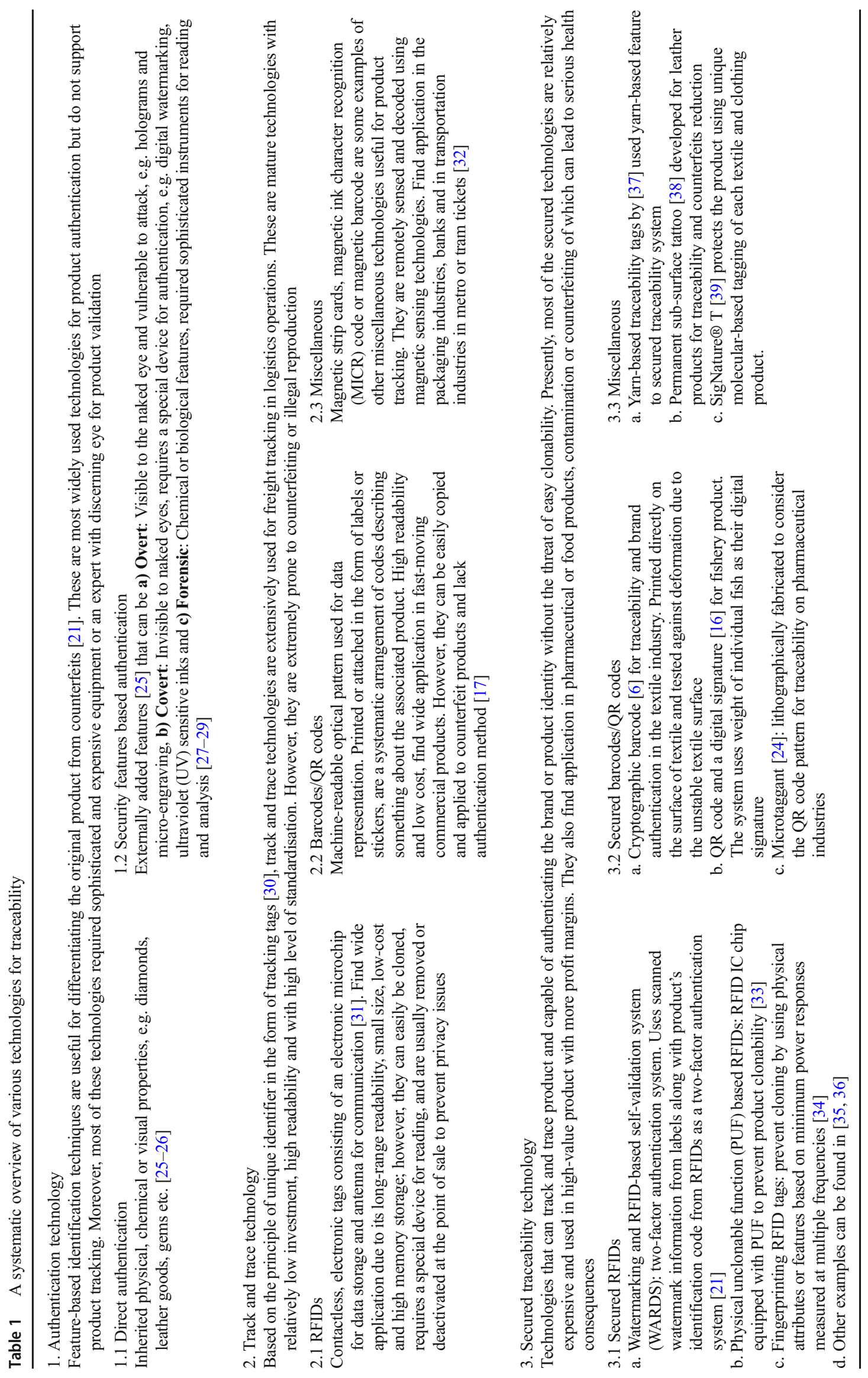




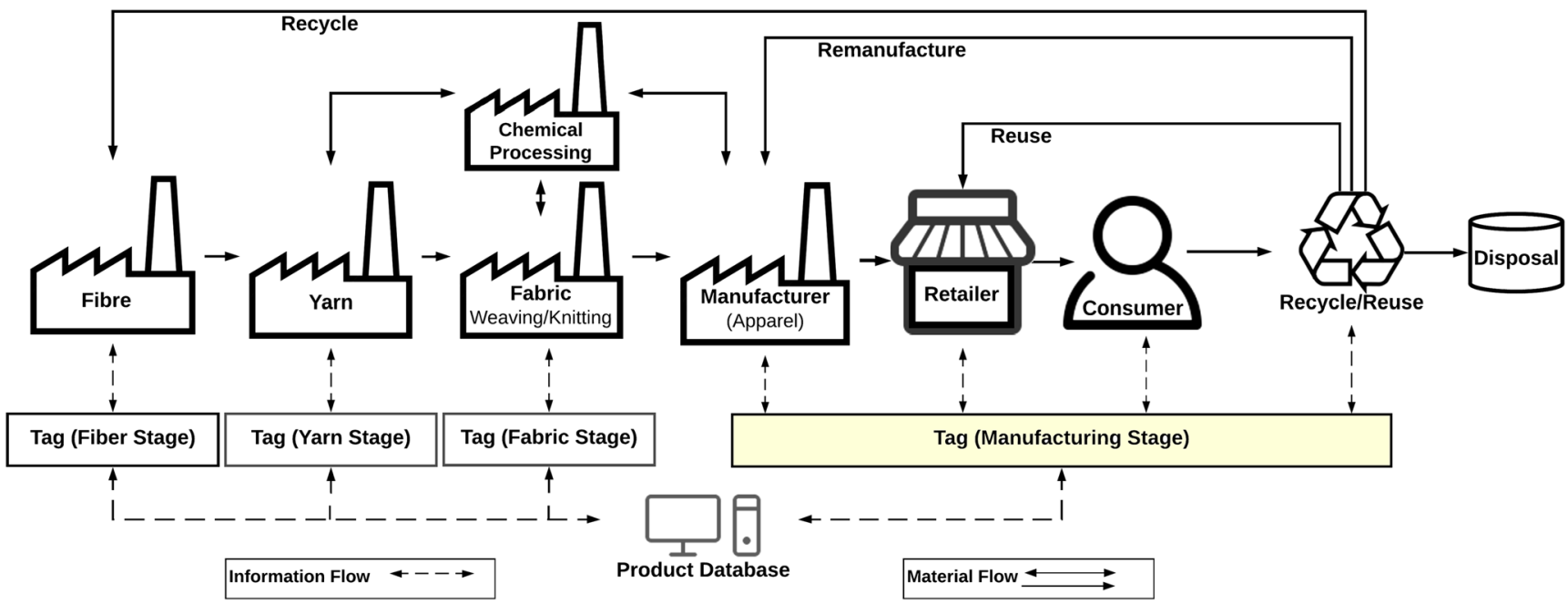

Fig. 1 An ideal secured traceability system for T\&C supply chain

mechanism preventing the easy and unauthorised reproduction of tags. However, at the same time, it should be durable to withstand mechanical agitation during washing and abrasion. It should also be integrated onto the flexible textile product to prevent the disintegration of tracking tag. Finally, taking into account the usual low cost of textile product, the developed solution should be inexpensive, eco-friendly and readable by a readily available device to allow the customer to authenticate and trace the product.

\section{Ideal secured traceability system}

As aforementioned, traceability is an item-centric approach tracking, tracing and connecting each physical product with their information. Within this context, traceability tag plays a crucial role in uniquely identifying the products and acts as an information-recalling agent. However, from a practical point of view, a tag also acts as the weakest point while implementing a secured traceability in the supply chain [23]. The whole traceability system can be compromised if the tag is copied, washed away or detached. In an ideal secured traceability system, each product should be tagged with a secured tag. These tags should be hard to replicate, easy to integrate in the product or developed using inherent features and should remain with the product throughout its life cycle. For such a system in $\mathrm{T} \& \mathrm{C}$ supply chain, since the material lots are combined and transformed into new materials with changed physical or chemical characteristics; therefore, a single tag may not be sufficient to cover the complete supply chain.

As shown in Fig. 1, these secured tags should be integrated with the product/intermediate product at each supply chain stage before it passes to the next downstream level. Thus, each tag acts as a link to access the product data management system, responsible for stacking data in a time-based function, as the product passes through different life cycle stages. This database system must retain and should include the data related to current stage and that received from previous stages. Finally, the
Fig. 2 Tag registration (mapping of code with QR code) and verification process

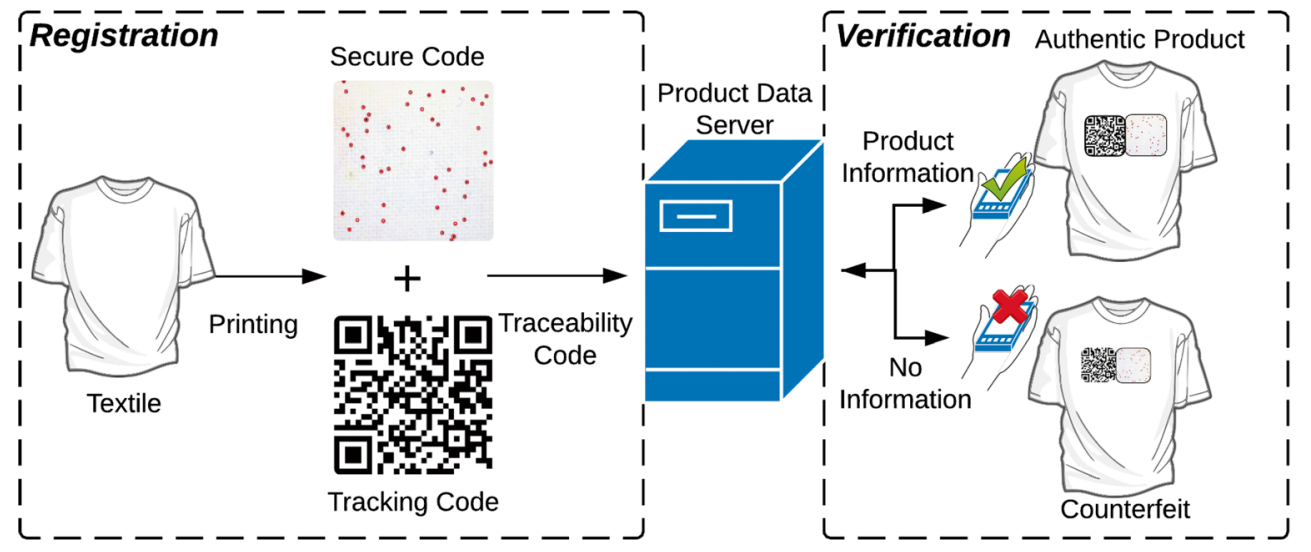


tag integrated at the final manufacturing stage should incorporate the manufacturing data and data from all previous stages.

The current study focuses on the secured tags that must be integrated on each textile product during the manufacturing (apparel production) stage before it passes to the retailer and finally to the customer. Design and concept of such integrated secured tags are presented in the following section.

\section{Concept and design}

\subsection{Concept}

According to Oxford Dictionary [41], security can be defined as 'procedures followed or measures taken to ensure the security of a state or organization'. One such measure to ensure the security of a physical entity consists of introducing features or markers that cannot be replicated or altered, and any attempt to tamper them leads to noticeable changes. These markers or features are usually introduced directly on the surface of the product through direct part marking (e.g. [42]) and act as a unique identifier to track and trace the product throughout the life cycle. Within this context, the study presents a novel methodology for the development of a secured tag or unique identifier for T\&C supply chain. To develop these, micro-sized particles are mixed with printing paste and then screenprinted on the surface of the fabric. The natural positioning of the particles on the fabric surface forms a random and unique distribution on each product. These random distributions are characterised and converted into security features. It primarily has two advantages. Firstly, the use of conventional screen printing (which is a mature manufacturing process with low capital cost and find wide applications in surface functionalization; for example, see [43]) and commercially available printing materials, which are widely used. Selection of such printing mechanism and material does not significantly influence the production process. It generates value from the existing technology with possible low implementation cost. Secondly, the position of the particles cannot be controlled during the printing process; therefore, practically, it is extremely difficult - if not impossible - to control the position of each individual particle while printing on the fabric surface. This provides an extraordinary advantage as no one can replicate the positioning of the particles through a controlled process, thus acts as a strong security feature. In order to implement it, the positioning of particles has to be mapped with 2D QR code, i.e. QR code would retain the information related to the positioning of the particles and the tracking code. As shown in Fig. 2, for verification, the information of particle positioning and tracking code associated with QR code should match with that of particle position printed on the textile surface and this correct mapping will only be possible for an authentic product.

It can be noted that the particle positioning can be either directly stored on the tag or indirectly on a server with reference to the QR code.

\subsection{Design}

The design of the secured tag is inspired from the coating Physical Unclonable Function (PUF) generated using explicitly introduced randomness. Widely used in the integrated circuit chip manufacturing industries to prevent counterfeits, these PUFs are easy to evaluate and read but hard to replicate [33]. The randomness acts as a fundamental building block of the cryptographic system and plays a crucial role in generating a strong secured communication key [44]. Therefore, randomness similar to PUFs is generated by scattering micro-particles in a confined area, on the surface of textile by an uncontrolled process. This type of security mechanism does not give control of code generation and security to a single trusted party or dependency on a single special product (e.g. special ink and microfiber); on the other hand, it would cast traceability onto the product (e.g. [45]). Therefore, the same tag cannot be reproduced even by the original manufacturer. Moreover, with an inconsistent concentration of the micro-size particle in relatively high tag area (in a few square centimetres), numerous tags can be printed with a very low probability of reproducing the same particle positioning again. The particle distribution on the secured tag is located using image-processing tools that are frequently used in surface classification and identification technologies (one such example can be found in [46]). Further, two distinct features (strong particle clusters and empty areas location, further explained in the 'Methodology' section) were extracted using particle locations and encoded to form the secure code. These innovative features take into account the flexible nature of textile surface, the durability concerns and also possible tampering of code through particle addition or erosion. Besides, use of image-based characterisation makes the secured tag readable by the smartphone-based camera, thereby giving customers the power to trace back the history of the product and making the supply chain more transparent and visible.

\section{Methodology}

The methodology followed in this study involves the printing of the secured particle-based tag (from hereafter referred as 'secured tag') on the textile surface, image of which is captured using smartphone, followed by processing of these images through image-processing algorithm to extract the tag features. Later, these features are registered, taking into account the flexible, unstable and non-uniform nature of textile surface, through a fuzzy membership function to get a code 
Fig. 3 Magnetic table and screen printing setup adopted in the current study

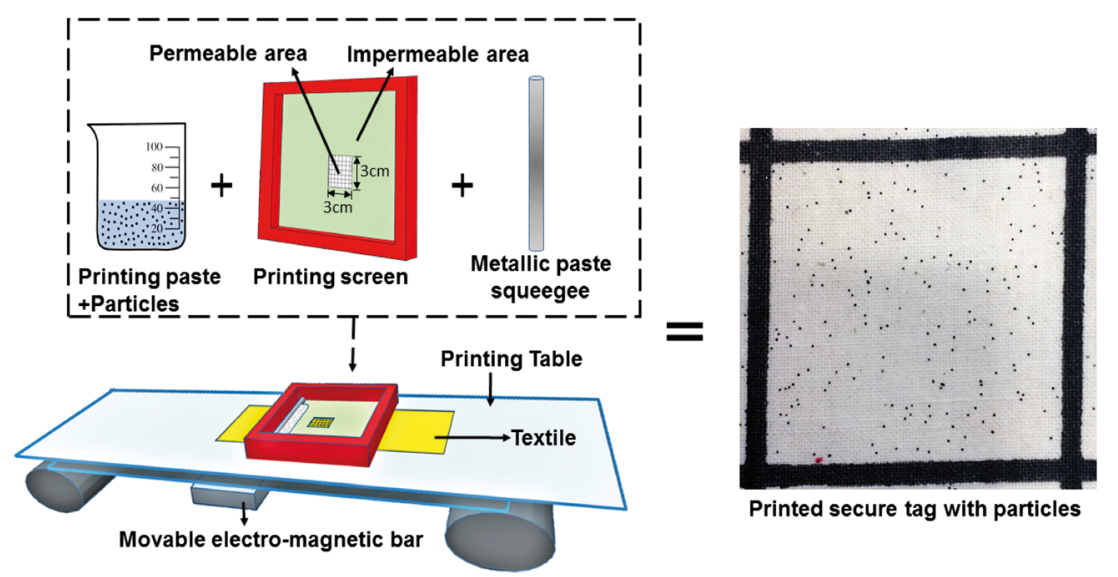

and mapped with the QR code. The current study focuses on particle-based secured tag, whereas printing of QR code is already a mature technology.

\subsection{Screen printing of secured tag}

Secured tag is integrated on the textile surface through screen printing mechanism and textile printing paste. Screen printing is a stencil printing method in which

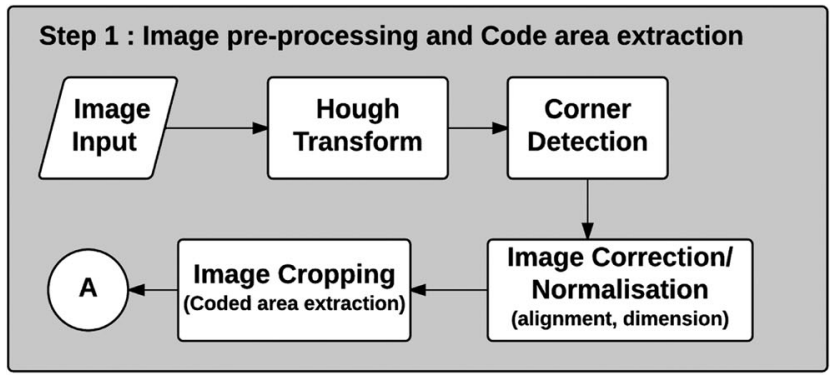

a

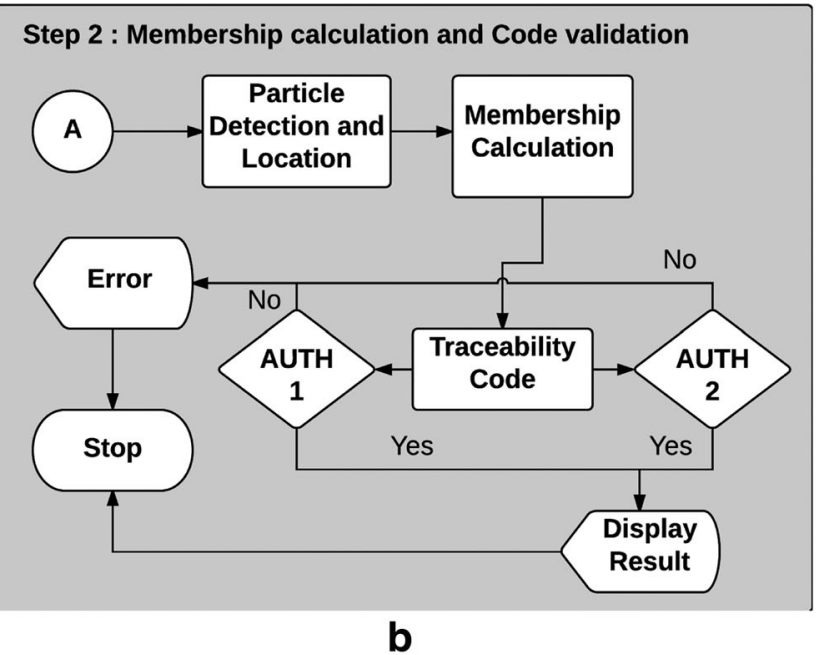

Fig. 4 Flow chart for tag encoding and validation mechanism. a Steps for image pre-process and code area extraction. b Steps for extracting the features, calculating the membership and validating the code printing paste or ink is squeezed through a fine mesh (made up of synthetic or metallic fibre) onto the fabric surface, except the areas that are made impermeable by the blocking stencil. The printing paste is formulated by mixing micro-sized dark glitter particles (sourced from Geotech International B.V., Netherlands) with a commercial textile binder in a fixed weight-by-volume ratio. First, a square frame of inner area $3 \mathrm{~cm} \times 3 \mathrm{~cm}$ was printed on the textile surface using black ink on a light-coloured fabric. (Relatively, a larger area of the tag will increase the possibility of numerous distributions and decrease the probability of generating same distribution). On the other hand, corner points of the black frame help in easy and fast detection of the quadrangles of the tag by referring to only a few pixels instead of the entire captured image [47]. It is also useful to determine the transformation caused by scaling, rotation and translation during the image-processing operations [48]. Printing paste (with micro particles) is then squeezed through the screen onto the fabric area inside the empty frame. The binder being almost transparent does not impart any colour to the light background; however, it strongly holds the particles onto the surface of the textile. This makes a good colour contrast that can easily be detected and captured in a digital image. For constant and uniform placement of the printing paste, an automatic squeezing mechanism was adopted using a magnetic table as shown in Fig. 3.

It should be noted, for commercial processes with large batch size, fully automated rotary screen printing can be adopted to reduce the process time.

\subsection{Tag encoding and validation mechanism}

As illustrated in Fig. 4, the tag encoding and validation mechanism followed mainly two steps. First set of steps were carried out to pre-process the image and extract the area of interest (particle area) followed by membership calculation (optical feature extraction and defining decision support system) and finally the validation step, where 
Fig. 5 Image obtained during different stages of encoding and validation process. a Original. b After correction, Otsu thresholding, and flood fill operations. c After Hough transformation. d Cropped image (area of interest). e Particle detection. f Particle location (after CHT)

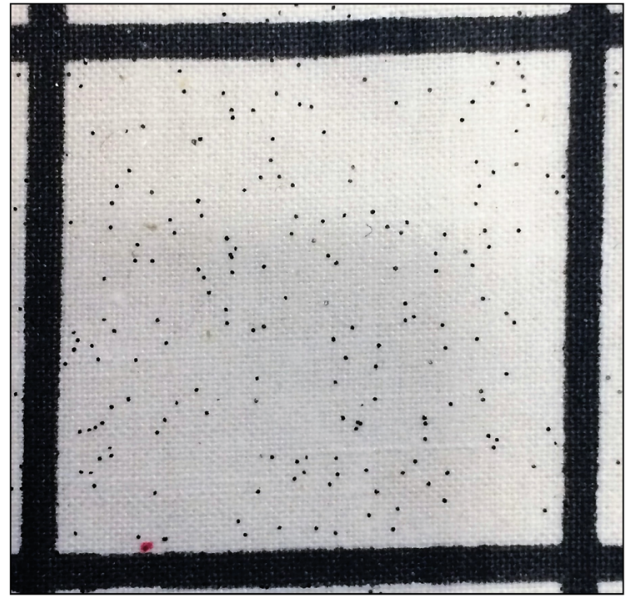

(a)

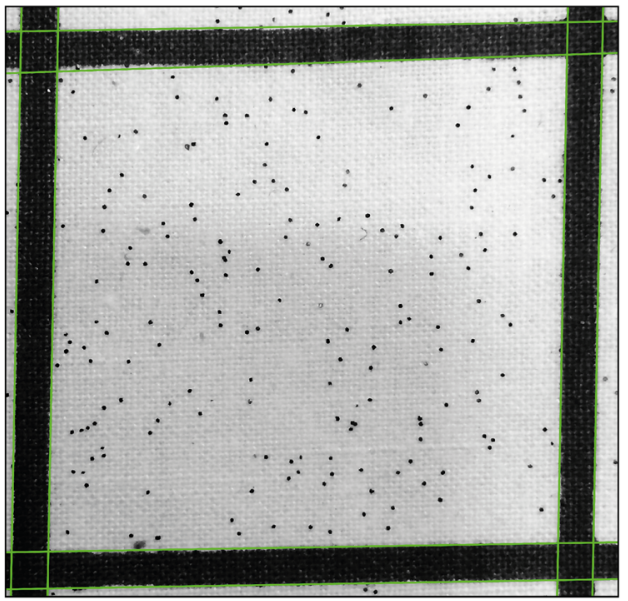

(c)

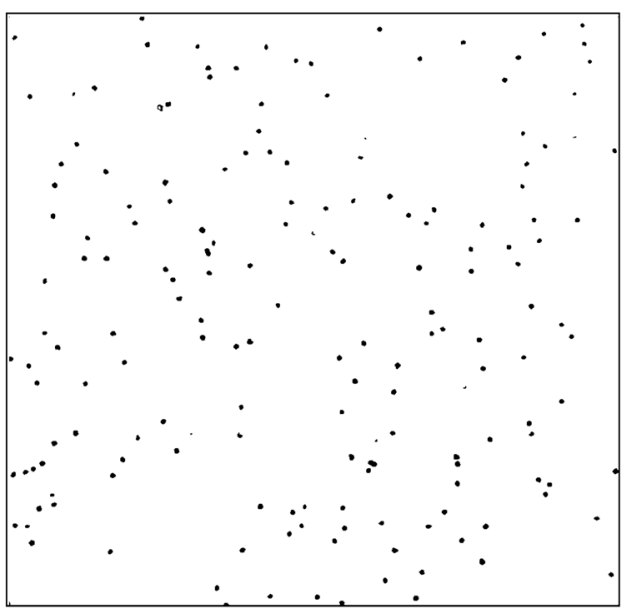

(e)

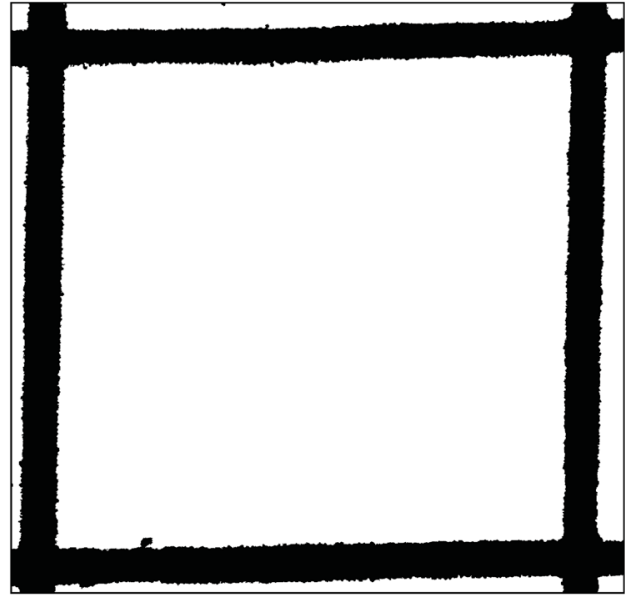

(b)

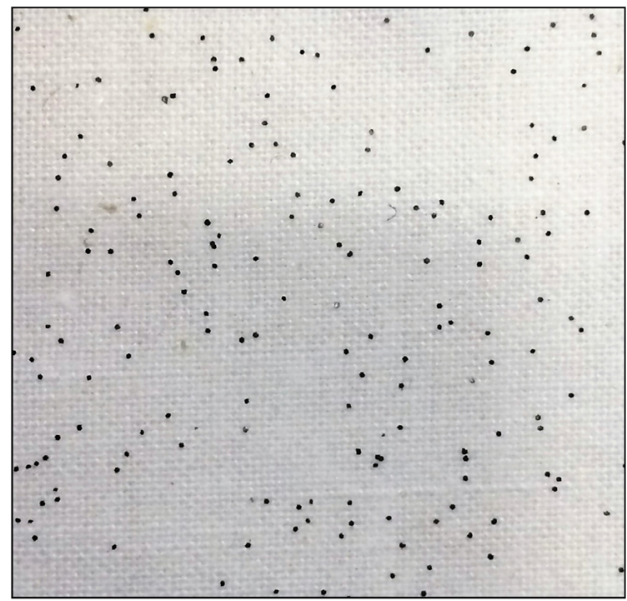

(d)

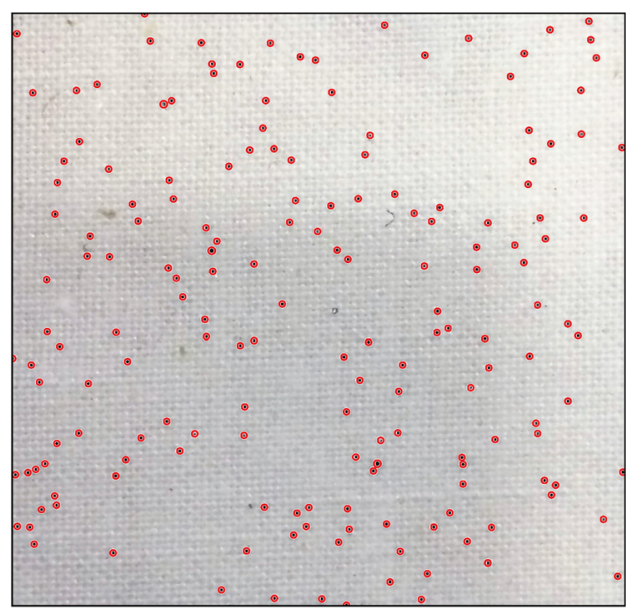

(f) the particle mapping (AUTH 1) is matched with QR code (AUTH 2) to authenticate and trace the product. It should be noted here that image pre-processing, code area extraction, particle detection and location steps would remain common for encoding and validation mechanism.

\subsubsection{Image pre-processing and code area extraction}

Image pre-processing is an important operation involving improvement of image data to suppress unwilling distortions and enhance image features that are important for further 
processing [49]. Mainly two groups of operations are applied to the captured image during this step. The first set of operations involved morphological operators and linear contract adjustment to correct the non-uniform illumination [49]. These are general methods applied by estimating the properties of degradation and evaluating image pixels during processing. The image was then converted to greyscale before further processing. The second group of operations was dedicated to detecting the quadrangle frame and its corner points followed by extraction of inner tag area printed with particle. For this, a copy of the processed greyscale image is converted to binary images using Otsu method (Otsu [50]). Otsu's method is a histogram threshold method that assumes the pixels of the image consist of classes, i.e. background and foreground, and calculate optimum threshold $t$ separating the two classes. Thus, the greyscale 2D image in Cartesian plane with coordinates $(x, y)$ and pixel intensity $I(x, y)$ is converted to binary image $B(x, y)$ with intensity 0 or 1 based on the derived threshold $t$ where,

$B(x, y)=\left\{\begin{array}{cc}0 & \text { if } I(x, y)<t \\ 1 & \text { otherwise }\end{array}\right.$

This was followed by flood fill method [51] to remove all the unwanted background (including the particles) to extract only the frame. Flood fill algorithm checks each pixel in an image and analyses the connected area. It removes any unwanted noise by filling/changing the intensity of the pixel to be the same as the intensity of the majority of neighbour. As a result, image in Fig. $5 \mathrm{~b}$ is obtained.

Further, Hough transformation [52] was applied to the obtained image to detect straight lines of the frame and thereafter the corner points, as shown in Fig. 5c. In Hough transformation, all the high-level pixels of a binary image are converted to polar coordinate $(r, \theta)$ form 2D Cartesian coordinate $(m, n)$ following relationship:

$r=m \cos \theta+n \sin \theta$

Such that $\theta$ is confined between $[0, \pi)$ and results in forming a unique Hough's peak/points on the polar plane for all mutually linear points in the Cartesian plane. However, in practice, due to geometric distortions, the location of the corner might change from initial to subsequent readings. Therefore, 2D projective transform [53] was performed along the detected corner points of the tag on the initial greyscale image to normalise it by correcting the alignment and scaling it down to a fixed dimension. Finally, the normalised image was cropped along the corner points to extract the actual area of interest, i.e. particle area as shown in Fig. 5d.

\subsubsection{Particle location detection}

After pre-processing operations, histogram-based intermode thresholding [54] was applied on the cropped image to extract the particle by subtracting the background (consisting of light colour textile), as illustrated in Fig. 5e. The input in this process was a greyscale image for which a histogram of the pixel intensity values (ranging from 0 to 255) was plotted and iteratively smoothed until only two peaks remained. The threshold value lies in the midpoint of the two peaks or the minimum point between the peaks corresponding to low-intensity white background pixels and high-intensity pixels with particles [55]. After identification of particles, in order to locate the center coordinates of particle, Circular Hough Transformation (CHT) [52] was applied on the obtained binary image. The resultant image is shown in Fig. 5f. CHT is similar to the standard Hough transform; however, the later tries and searches for circles in the given image between a predefined range of radius. Due to this predefined radius range (derived from calculating the average particle size and standard deviation), it is easy for the algorithm to differentiate two or more close or connected particles.

\subsubsection{Membership calculation}

After determining the location of each particle, two key features were extracted. The first key feature was the location of the strong clusters of the particles. In other words, areas in the tag where particles are very close to each other having a strong degree of belongingness in the cluster. The second feature was the locations of empty areas in the tag, especially the largest empty areas. It should be noted that these two features are inter-dependent. Any attempt to copy the features is made by increasing or decreasing the concentration of particles in the tag, one of the two features will vary significantly. There should be a balance between the two features that has a high probability to be present only in the original/authentic tag.

\section{Locating strong clusters of particle}

Initially, all the particle locations (observations) were divided into $k$ predefined optimum clusters based on their location (Euclidean distance) using $k$-means clustering algorithm described below.

$$
\begin{aligned}
& \operatorname{argmin} s \sum_{j=1}^{k} \sum_{x \in S_{j}}\left\|x-c_{j}\right\|^{2}=\arg \min s \sum_{j=1}^{k}\left|S_{j}\right| \operatorname{Var} S_{j} \\
& \operatorname{argmin} s \sum_{j=1}^{k} \frac{1}{2\left|S_{j}\right|}=\sum_{x, y \in S_{j}}\|x-y\|^{2} \\
& \sum_{x \in S_{j}}^{k}\left\|x-c_{j}\right\|^{2}=\sum_{x \neq y \in S_{j}}\left(x-c_{j}\right)\left(c_{j}-y\right)
\end{aligned}
$$

where $x=$ observation (particle coordinates), $c_{j}=$ centroid for the cluster $j$ and $S_{j}=$ set of particle location in $j$ cluster. 
The value of $k$ is obtained through Elbow method. During clustering, given set data is divided into groups of similar elements; however, there are set of data that have high fuzziness or uncertainty of belongingness to allotted cluster/group. Similarly, in case of $k$-means clustering, the data points on the edge of the clusters will have a high probability of lying in the neighbourhood cluster. Hence, it becomes an important criterion to locate and store the strong clusters removing out all the outliers. Therefore, to eliminate all the outliers or points with high uncertainty of belongingness, the following steps were taken:

- Data from each obtained clusters were segregated and treated separately.

- Euclidean distance between each data point and cluster centroid was calculated.

- Area of the largest circle with the centre as the centroid of the cluster and radius equal to the distance of most distant particle in the same cluster was calculated.

- Graph of percentage increases in the area of the circle (from 0 to $100 \%$, i.e. the area of the largest circle, at an interval of 10) against a cumulative number of particles within given area was plotted.

- The bending (elbow) point of the curve was calculated and the percentage area against it became the threshold area for the new cluster. Value of radius corresponding to the threshold area became the new largest radius.

- The centroid of the previous cluster later shifted to the centroid of the new cluster with reduced points.

- The radius and centres of all the $k$ cluster were selected as part of the code.

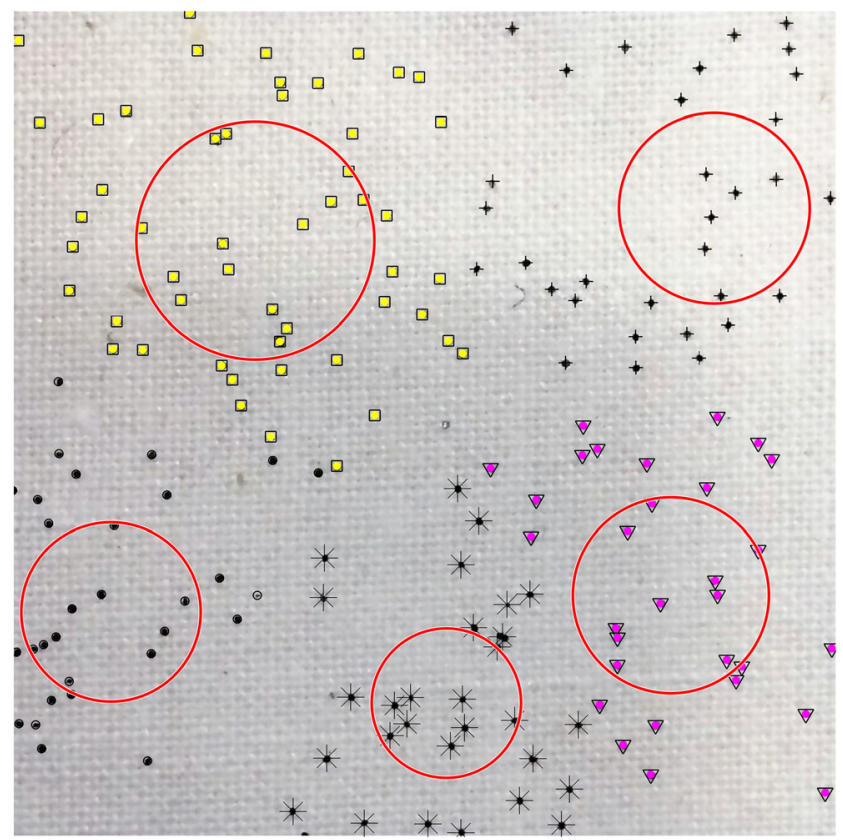

Fig. 6 Particles in different clusters marked with different symbols with circle enclosing the newly formed cluster
Abovementioned steps were applied on the particle locations obtained from the image in Fig. 5f. These locations were divided into five clusters (marked with different symbols in Fig. 6), and later five strong clusters were extracted (encircled in Fig. 6).

The main reason for considering the location of strong particle cluster as an encoding feature is to avoid dependency on the location of a single particle. Therefore, even if some are eroded or added (noise), the average membership of the particles in the respective cluster should not vary significantly.

\section{Locating empty areas}

A more logical and systematic approach of locating and encoding empty area is to find the largest circle which can fit in that empty area without any particle inside. For this, Voronoi diagram and Delaunay triangulation methods were opted [56]. Delaunay triangulation, which is a popular computational geometry method of subdividing a given 2D Euclidean plane with a finite number of points in it into triangles such that none of the points on the given Euclidean plane, lies inside the circumcircle of any triangle. Voronoi diagram of a set of points is dual to its Delaunay triangulation.

For $K$ different points in the 2D space, $R^{2}$ the Voronoi cell $V_{k}(i)$ of $k_{i}$ is denoted by:

$V_{k}(i)=\left\{q \in R^{2} \mid\left\|q-k_{i}\right\| \leq\|q-k\|\right.$ for all $\left.k \in K\right\}$

After applying Delaunay triangulation, radiuses and centres of all the distinct circumcircles with no overlapping areas

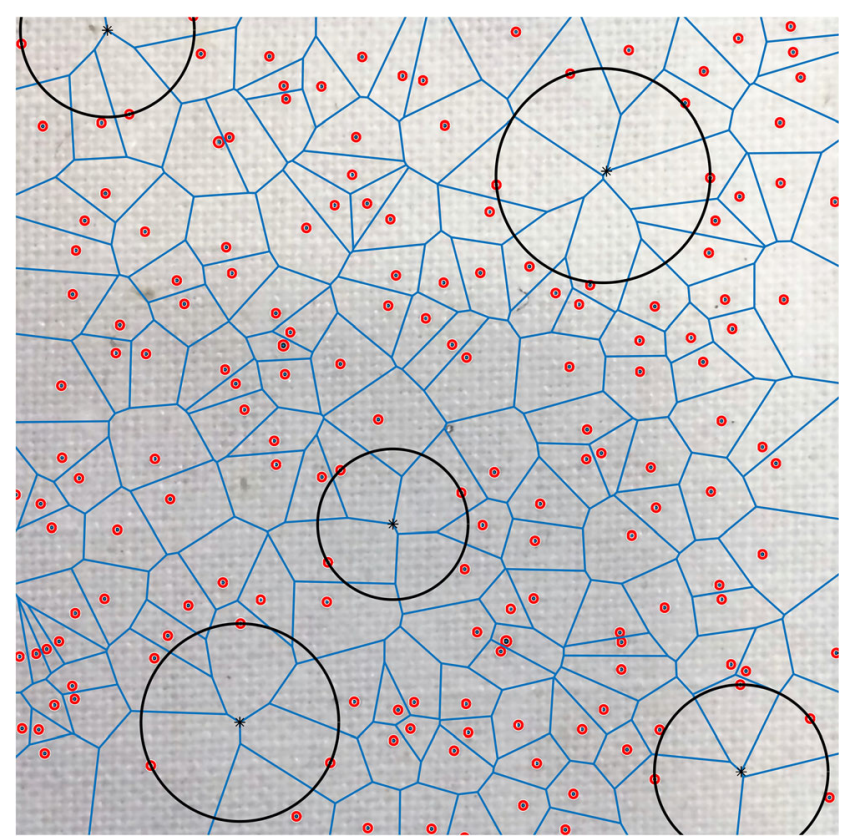

Fig. 7 Partition of the image plane into Voronoi cells and five largest circles, encircling the five biggest empty spots in the image 
were recorded and top $k$ largest circles (same as the optimum number of clusters obtained through Elbow method) were selected from them as a part of the optical feature. Figure 7, illustrates the application of Voronoi diagram on the particle locations obtained from the image in Fig. $5 \mathrm{f}$ and location of five biggest empty areas encircled in Fig. 7.

\subsubsection{Decision support system for tag feature encoding}

For an effective and fast decision support system (DSS) algorithm, it is not necessary to match exactly the true pattern with subsequent reading; rather, it should compute the difference between distributions of correlated like pattern with that of unlike pattern. Moreover, taking into account the flexible, non-uniform and uneven textile surface, while defining a membership function, it is necessary to incorporate some tolerance. In this direction, fuzzy set principle introduced by Zadeh [57] using the concept of similarity of inputs instead of crisp values were used to define and assign a fuzzy membership function to these features. Membership function $f(x)$ is a function that assigns, to every number $x$, the degree $f(x)[0 ; 1]$ to what this number satisfies the corresponding property. For example, in the proposed secured tag, the degree to which the obtained feature will belong to the original code while decoding. For the current study, one of the most frequently used trapezoidal membership function was selected. As described in the previous section, the location particles of the strong cluster and the empty areas are encircled and referred by the centre $(c)$ and radius $(r)$ of the circle. Therefore, the trapezoid membership $f(x)$ is defined as:

$f(x)=\left\{\begin{array}{cc}0 & \text { if } x>r \\ 1 & \text { if } x<\frac{r}{2} \\ -\frac{2 x}{r}+2 & \text { otherwise }\end{array}\right.$

where $x$ is calculated as the Euclidean distance of the particle from the centroid of the cluster, i.e.

$x=\sqrt{\left(a_{0}-a\right)^{2}+\left(b_{0}-b\right)^{2}}$

such that $\left(a_{0}, b_{0}\right)$ are the coordinates of the cluster centroid and $(a, b)$ are the position of a particle. Since there are more than one particle in each cluster, the average membership of the cluster $(\mu)$ is calculated as:

$\mu=\frac{\sum_{i=1}^{n} f\left(x_{i}\right)}{n}$

where $n$ is the total number of particles and $f\left(x_{i}\right)$ is the membership to the $i^{\text {th }}$ particle in the cluster. Moreover, since each tag consists of multiple clusters, the membership of all clusters can be represented as $\mu_{i}, i \in\{1, k\}$ where $k$ is the total number of clusters. In order to increase the robustness of DSS and avoid ambiguity and uncertainty, individual membership of two optical features extracted in the previous section was aggregated. For the first feature, membership of each particle as per their location in the respective cluster was calculated and average membership values of each cluster were added to get the final membership of the first feature (strong clusters). For the second feature (empty area), the initial membership value will remain zero, as there will be no particle inside while encoding. However, while decoding, the membership value may vary due to deformation.

\subsubsection{Membership difference for code validation}

The membership difference is calculated as:

$d=\sum_{i=1}^{k}\left|\mu_{i}^{o}-\mu_{i}^{T}\right|$

Such that $\mu_{i}^{o}$ represents the membership of the $i^{\text {th }}$ cluster for the original tag and $\mu_{i}^{T}$ represents the membership of the $i^{\text {th }}$ cluster of the tested tag. It should be noted that the coordinate points of the clusters and their average membership value of the original tag should be stored in order to make a comparison as shown in Fig. 8.

\section{Experimental}

As mentioned before, in practice, unavoidable variations can occur in the initial and subsequent reading of the tag due to variations in camera, image resolution, light intensity, light temperature, etc. The most common variations that can occur in such textile-based tags are particle erosion due to washing or abrasion during textile use phase, pattern deformation due to elastic nature of textiles, noise (in form of migrated particle or foreign particle/lint) and alignment variation due to inappropriate handling of the camera while decoding. Therefore, to test the developed system for these variations, 50 different tags were printed. Images of these tags were captured with

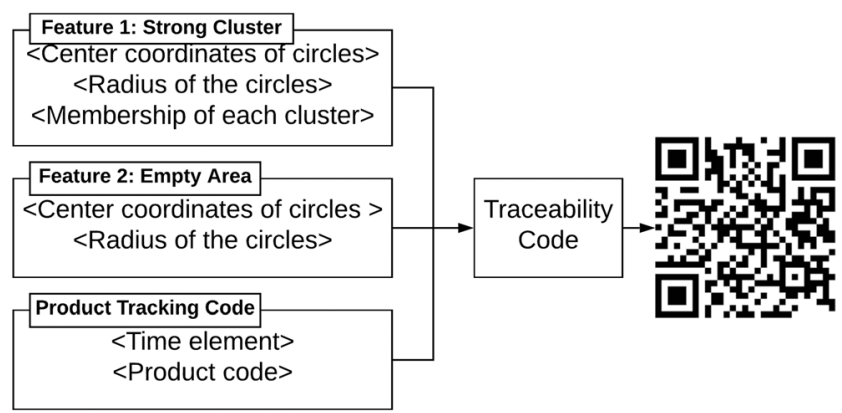

Fig. 8 Different parts of the traceability code that would be encoded and printed as QR code on the textile surface 
Fig. 9 Variation of membership difference for inter-tag analysis

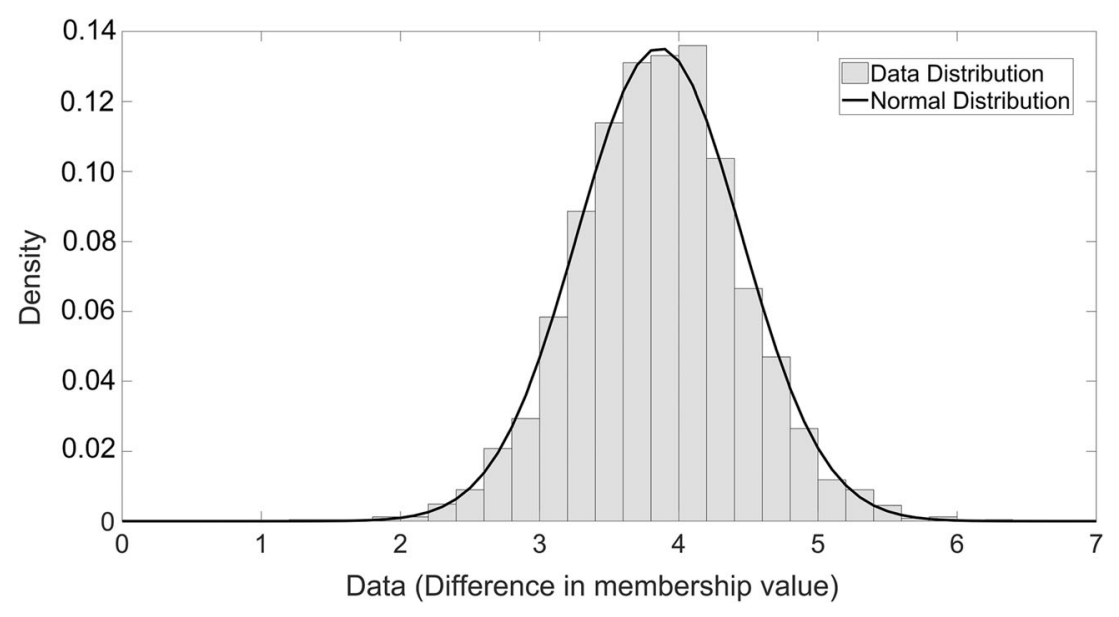

digital camera and processed using MATLAB 2015b as per the methodology explained in the 'Ideal secured traceability system' section. Variations were introduced synthetically followed by image decoding operation to verify the tag identity. Results obtained after decoding each tag were compared with the actual authentic code and with other 49 codes of different tags to validate the code and test the randomness.

\subsection{Inter-tag analysis}

An initial experiment was conducted to prove the uniqueness of each tag and estimate an average membership difference between two unlike tags. Membership code of each tag was applied on itself and on the other 49 tags. Frequency distribution of membership difference of 2500 possible combination thus obtained was plotted, to estimate the average and standard deviation value.

\subsection{Alignment analysis}

The tags are unidirectional and encoded in one fixed alignment. During verification, the algorithm would check the image by aligning and decoding it in all four directions, i.e. 0, 90, 180 and 270 (alternately, a positioning marker can be added like the QR code). The code should only validate with the original authentic code in one alignment and there should be variation in membership value in all other direction. Code of the authentic tag was compared with decoded images of 50 tags by rotating them in all directions. The experiment was repeated for all the 50 codes, and 50 images. The differences thus obtained were recorded.

\subsection{Particle addition/erosion analysis}

The proposed textile tag should be durable to withstand mechanical agitation. Washing and abrasion during the regular
Fig. 10 Difference in membership value of the original tag and membership obtained when it is rotated by $\mathbf{a} 0^{\circ}, \mathbf{b} 90^{\circ}, \mathbf{c}$ $180^{\circ}$, and $\mathbf{d} 270^{\circ}$ (a)




Fig. 11 Difference in membership value when particles are added or removed

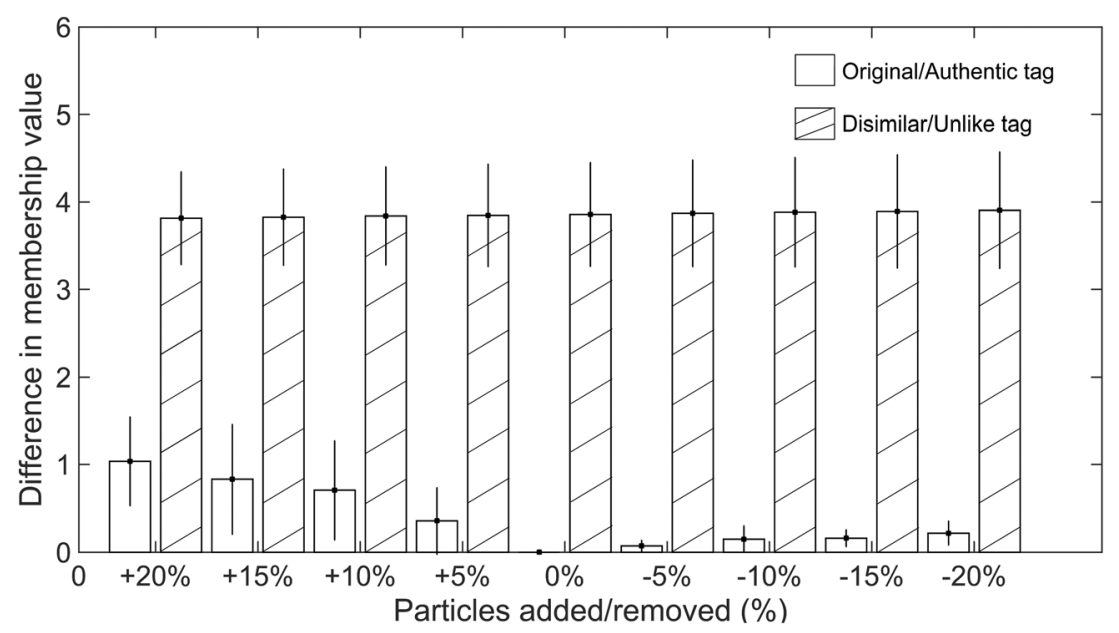

use of the $\mathrm{T} \& \mathrm{C}$ product can erode some particles or add some unwanted noise in the tag. To simulate the same, up to $20 \%$ of the particle locations were randomly added and removed in the obtained particle locations in original image and results were analysed in the subsequent section.

\subsection{Pattern distortion analysis}

Due to flexible nature of textiles, the original tag might stretch in due course of usage. In order to simulate such conditions, the original tag images were stretched to $20 \%$ in vertical, horizontal and diagonal direction and realigned about the corners using projective transform.

\subsection{Particle shift}

Due to improper image capturing method or shift in corner points, the particle location might shift to a certain extent. To simulate the same, all the obtained particle locations from the original tags were rotated up to $10^{\circ}$, leading to a rotational and linear shift. The obtained locations were then validated to check the difference.

\section{Results}

For a robust, secured traceability system, the tags should be durable to last until the recycling stage. Pertaining to this, the experiments analysed the tag performance under common variations that might affect the subsequent tag reading due to its textile nature. The results are presented in terms of membership difference of 50 tags. To ensure low probability of false detection, the average value of membership difference between like patterns of the authentic tags before and after variation should be zero or very low, whereas the average difference between two unlike pattern should be high enough to result in significant and complete separation to facilitate selection of a threshold value. All the difference value below the threshold will have a high probability that the read pattern
Fig. 12 Difference in membership value when the tags are stretched to $20 \%$ in a horizontal, b vertical, and c diagonal directions

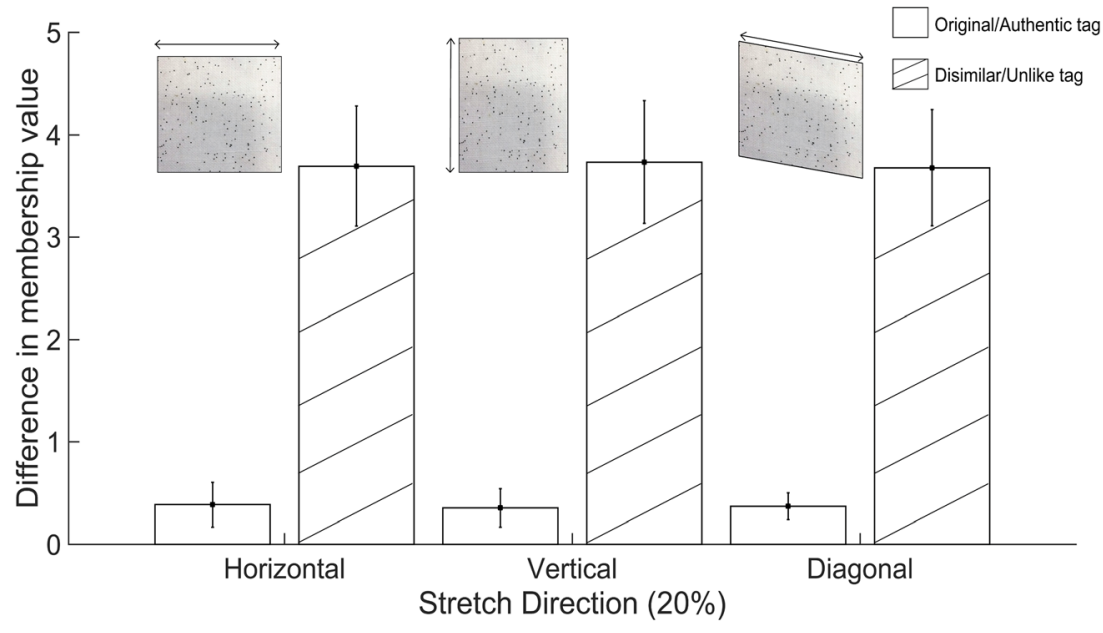


corresponds to the original tag even after variation, whereas the difference value above threshold corresponds to unlike or unauthentic code.

\subsection{Results for inter-tag analysis}

The frequency distribution of the membership difference for all the comparisons was plotted. It was observed that it followed a normal distribution, as shown in Fig. 9.

The average membership difference among two unlike tag was 3.89 with 0.59 standard deviation. It should be noted that membership difference for the tag, when compared with itself for all the 50 cases, came out to be zero and all those values were neglected for the distribution evaluation.

\subsection{Results for alignment analysis}

Figure 10 shows the average difference in membership value obtained by subtracting the original or authentic tag membership value with the correctly aligned original tag, followed by average difference between the membership value of authentic tag and membership obtained after rotating the same with $90^{\circ}$ subsequent rotation. A complete and vast separation can be observed between the correctly aligned and misaligned tag, proving that the pattern is random and will authenticate only with correct alignment and correct pattern.

\subsection{Results for particle addition/erosion analysis}

Figure 11 shows the results of the average difference in membership values obtained after one-to-one comparison between first the distorted pattern with the authentic tag patterns and then with pattern of the 50 unlike tags. These distorted patterns are obtained after randomly removing or adding particle location values up to $\pm 20 \%$. It can be observed that the membership difference for the like or authentic tags with distorted pattern increases with increasing the level of erosion or addition. However, the difference between the unlike tags membership value is still high that proves the probability of false detection is still low even after removing or adding up to $20 \%$ of particles.

\subsection{Results for pattern distortion analysis}

All the 50 patterns were stretched to $20 \%$ horizontally ( $x$-direction), vertically ( $y$-direction) and diagonally (in $45^{\circ}$ direction) and realigned using proposed methodology.

Figure 12 shows the mean difference in membership values of original/authentic tags with undistorted patterns and that obtained after realigning the like pattern on one side. This is followed by mean difference in membership values of the realigned tag with other 50 tags on other. The results show a significant variation among the differences of like tags and differences of two unlike tags even after realignment and pattern distortion. This verifies that the algorithm proves effective even after stretching the tags and tolerates pattern distortion to a certain extent.

\subsection{Results for particle shift}

Figure 13 shows the mean difference in membership values of authentic/original tag with undistorted patterns and that obtained after shifting/rotating the locations of all the particle of the like pattern up to $10^{\circ}$ on one side and the average difference in membership values of the shifted pattern tag with other 50 tags on the other. It can be observed that the difference up to $5^{\circ}$ is not very high and the probability of false detection is comparatively low. However, a greater distortion more than $7.5^{\circ}$ can affect the algorithm and increase the probability of false detection. This factor can be studied further and improved in the future study.
Fig. 13 Difference in membership value with particle rotational shift

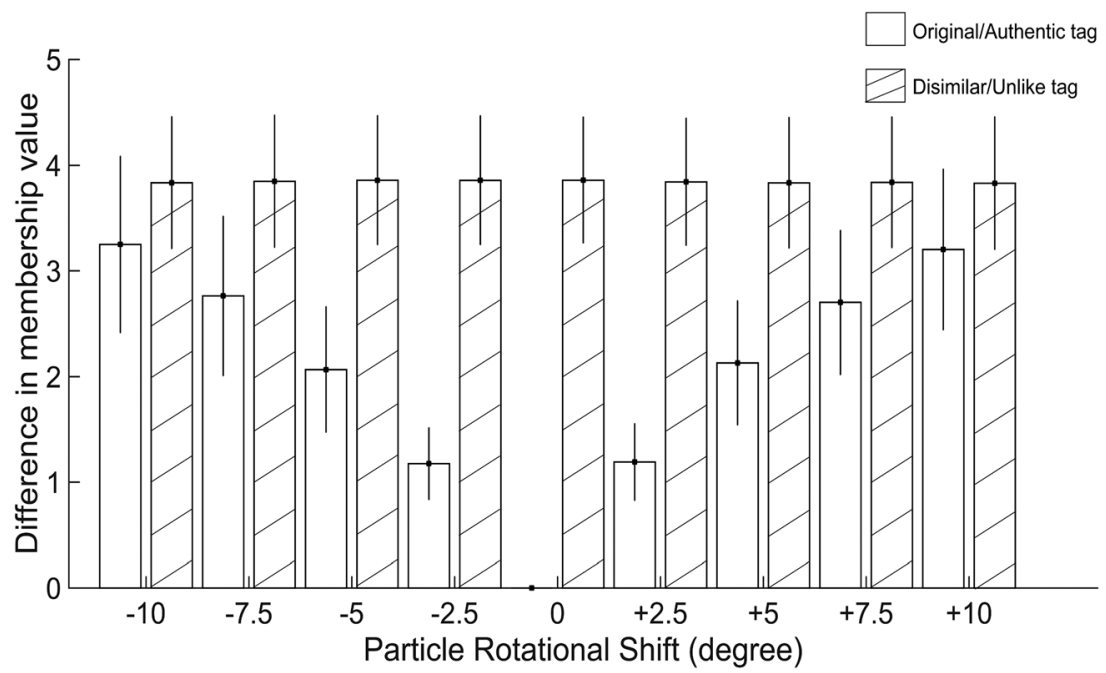




\section{Conclusions}

This paper introduces and validates concept, design and methodology of an integrated and secured traceability tag for the $T \& C$ supply chain. The main novelty and contribution in this work are (1) in the form of a secured tag specially designed and developed for the T\&C supply chain, taking into account the particularities of the $\mathrm{T} \& \mathrm{C}$ product, (2) in terms of value generation from the existing conventional printing technology and material with low capital investment and (3) selection and encoding of distinct features that complement each other and lead to high probability of true positive validation of the secured tag. It is anticipated that such tag would make the supply chain traceable and would be helpful in anti-counterfeiting. The developed tag showed good results when tested for use-phase treatment of the textile product. The tag only validates in one alignment, which proves that the distribution is random and unique. There was not a significant change in the membership difference value even after addition or removal of around $20 \%$ of particles. In addition, the membership difference did not increase (within the authentic tag) even after shape distortion due to stretching (up to 20\%). This is an important criterion for the durability of the tag, as it should be validated (if authentic) even after some distortion due to washing or handling process.

However, it has certain limitations that need be addressed in future research works. A physical wash and abrasion resistance analysis should be done to examine the impact of washing and abrasion of the tag under standard test conditions. It should also be noted the difference in membership value (for original tag) increased with the particle rotational shift. Therefore, the validation process can be affected by this distortion. An in-depth analysis of various commercially available textile binders can be done in future research work to restrict particle movement over the textile surface. This can be followed by piloting of the developed tag in the actual $\mathrm{T} \& \mathrm{C}$ supply chain to find and address the implementation challenges.

These secured tags presented in this study are helpful in securing the T\&C supply chain at the product level and restrict unauthorised access to the product database. Nonetheless, future research can be undertaken to secure the traceability system at the business level to prevent the attack on the information sharing mechanism.

Acknowledgments This work has been comprehended in the framework of Erasmus Mundus Joint Doctorate Project-Sustainable Management and Design for Textiles (SMDTex), which is financed by the European Commission. The authors would also like to thank Mr. Christian Catel from ENSAIT/GEMTEX, France for his time and help.

Open Access This article is distributed under the terms of the Creative Commons Attribution 4.0 International License (http:// creativecommons.org/licenses/by/4.0/), which permits unrestricted use, distribution, and reproduction in any medium, provided you give appropriate credit to the original author(s) and the source, provide a link to the Creative Commons license, and indicate if changes were made.

Publisher's Note Springer Nature remains neutral with regard to jurisdictional claims in published maps and institutional affiliations.

\section{References}

1. Jd M (2001) The importance of traceability for public health and consumer protection. Rev Sci Tech Int Off Epizoot 20:363-371

2. Myae AC, Goddard E (2012) Importance of traceability for sustainable production: a cross-country comparison. Int J Consum Stud 36:192-202. https://doi.org/10.1111/j.1470-6431.2011.01084.x

3. Kumar V, Agrawal TK, Wang L, Chen Y (2017) Contribution of traceability towards attaining sustainability in the textile sector. Text Cloth Sustain 3:5. https://doi.org/10.1186/s40689-017-0027-8

4. Kumar V, Koehl L, Zeng X (2016) A fully yarn integrated tag for tracking the international textile supply chain. J Manuf Syst 40:7686. https://doi.org/10.1016/j.jmsy.2016.06.007

5. Lee S-Y (2008) Drivers for the participation of small and mediumsized suppliers in green supply chain initiatives. Supply Chain Manag Int J 13:185-198. https://doi.org/10.1108/ 13598540810871235

6. Corbellini S, Ferraris F, Parvis M (2006) A cryptographic system for brand authentication and material traceability in the textile industry. In: 2006 IEEE Instrumentation and Measurement Technology Conference Proceedings. pp. 1331-1335.

7. McMillen D, Alvarez M, Craig S (2016) Security trends in the manufacturing industry [White Paper]. IBM, New York, USA

8. Wajsman N, Burgos CA, Davies C (2015) The economic cost of IPR infringement in the clothing, footwear and accessories sector. Office for Harmonisation in the Internal Market, Alicante, Spain.

9. Ekwall D ((2009)) The risk for detection affects the logistics system setup for cargo smugglers. In: Nofoma. Jönköping, Sweden. Jönköping, Sweden, p 2009

10. He W, Zhang N, Tan PS, et al. (2008) A secure RFID-based track and trace solution in supply chains. In: 2008 6th IEEE International Conference on Industrial Informatics. pp. 1364-1369.

11. Azuara G, Luis Tornos J, Luis Salazar J (2012) Improving RFID traceability systems with verifiable quality. Ind Manag Data Syst 112:340-359. https://doi.org/10.1108/02635571211210022

12. Saak AE (2016) Traceability and reputation in supply chains. Int J Prod Econ 177:149-162. https://doi.org/10.1016/j.ijpe.2016.04.008

13. Ilie-Zudor E, Kemény Z, Ekárt A et al (2014) A solution for information management in logistics operations of modern manufacturing chains. Procedia CIRP 25:337-344. https://doi.org/10.1016/j. procir.2014.10.047

14. Juels A (2006) RFID security and privacy: a research survey. IEEE J Sel Areas Commun 24:381-394. https://doi.org/10.1109/JSAC. 2005.861395

15. Jourová V (2017) Rapid alert system for dangerous products - annual report 2016. Publ Off Eur Union 2017:1-32. https://doi.org/10. 2838/069613

16. Seino K, Kuwabara S, Mikami S, et al. (2004) Development of the traceability system which secures the safety of fishery products using the QR code and a digital signature. In: OCEANS ' 04. MTTS/IEEE TECHNO-OCEAN '04. pp. 476-481.

17. Várallyai L (2012) From barcode to QR code applications. Agrár Agric Inform 3:9-17. https://doi.org/10.17700/jai.2012.3.2.92

18. Pigni F, Crave S, Ravaini A ((2007)) Traceability in the textile and clothing industry: issues and implications for RFId adoption. In: 
The 2nd Mediterranean Conference on Information Systems, MCIS. Venice, Italy, p 2007

19. Kang Y-S, Lee Y-H (2013) Development of generic RFID traceability services. Comput Ind 64:609-623. https://doi.org/10.1016/j. compind.2013.03.004

20. Cheung HH, Choi SH (2011) Implementation issues in RFID-based anti-counterfeiting systems. Comput Ind 62:708-718. https://doi. org/10.1016/j.compind.2011.04.001

21. Ting SL, Tsang AHC (2013) A two-factor authentication system using radio frequency identification and watermarking technology. Comput Ind 64:268-279. https://doi.org/10.1016/j.compind.2012.11.002

22. Burnotte L, Emmerecht E, Delaigle J-F et al (2006) Traceability rules against counterfeiting for international normalization groups. Belgium Science Policy. In: Brussels

23. Kumar V (2017) Exploring fully integrated textile tags and information systems for implementing traceability in textile supply chains (PhD Thesis). University Lille 1 in collaboration with Högskolan i Borås (Sweden) and Soochow university (Suzhou).

24. Han S, Bae HJ, Kim J et al (2012) Lithographically encoded polymer microtaggant using high-capacity and error-correctable QR code for anti-counterfeiting of drugs. Adv Mater 24:5924-5929. https://doi.org/10.1002/adma.201201486

25. Bansal D, Malla S, Gudala K, Tiwari P (2013) Anti-counterfeit technologies: a pharmaceutical industry perspective. Sci Pharm 81:1-13. https://doi.org/10.3797/scipharm.1202-03

26. Baldini G, Fovino IN, Satta R, et al. (2015) Survey of techniques for the fight against counterfeit goods and Intellectual Property Rights (IPR) infringement. Publ Off Eur Union 1-130. doi: https://doi.org/ $10.2788 / 186725$ (print)

27. Lei P, Claret-Tournier F, Chatwin C, Young R (2005) A secure mobile track and trace system for anti-counterfeiting. In: 2005 IEEE International Conference on e-Technology, e-Commerce and e-Service. pp. 686-689.

28. Yoon B, Lee J, Park IS et al (2013) Recent functional material based approaches to prevent and detect counterfeiting. J Mater Chem C 1: 2388-2403. https://doi.org/10.1039/C3TC00818E

29. Cui Y, Phang IY, Lee YH et al (2015) Multiplex plasmonic anticounterfeiting security labels based on surface-enhanced Raman scattering. Chem Commun Camb Engl 51:5363-5366. https://doi. org/10.1039/c4cc08596e

30. Ilie-Zudor E, Kemény Z, van Blommestein F et al (2011) A survey of applications and requirements of unique identification systems and RFID techniques. Comput Ind 62:227-252. https://doi.org/10. 1016/j.compind.2010.10.004

31. Nath B, Reynolds F, Want R (2006) RFID Technology and applications. IEEE Pervasive Comput 5:22-24. doi: https://doi.org/10. 1109/MPRV.2006.13

32. Sparavigna A (2008) Labels discover physics: the development of new labelling methods as a promising research field for applied physics. ArXiv Prepr ArXiv08012700.

33. Devadas S, Suh E, Paral S, et al. (2008) Design and implementation of PUF-based "unclonable" RFID ICs for anti-counterfeiting and security applications. In: 2008 IEEE International Conference on RFID. pp. 58-64.

34. Periaswamy SCG, Thompson DR, Di J (2011) Fingerprinting RFID tags. IEEE Trans Dependable Secure Comput 8:938-943. https:// doi.org/10.1109/TDSC.2010.56

35. Rieback MR, Crispo B, Tanenbaum AS (2006) The evolution of RFID security. IEEE Pervasive Comput 5:62-69. https://doi.org/10. 1109/MPRV.2006.17

36. Pateriya RK, Sharma S (2011) The evolution of RFID security and privacy: a research survey. In: 2011 International Conference on Communication Systems and Network Technologies. pp. 115-119.

37. Kumar V, Koehl L, Zeng X, Ekwall D (2017) Coded yarn based tag for tracking textile supply chain. J Manuf Syst 42:124-139. https:// doi.org/10.1016/j.jmsy.2016.11.008
38. Cataldo A, Grieco A, Prete AD et al (2016) Innovative method for traceability of hides throughout the leather manufacturing process. Int J Adv Manuf Technol 86:3563-3570. https://doi.org/10.1007/ s00170-016-8489-4

39. Hurry SM SigNature ${ }^{\circledR} T$ tagging and testing platform. In: Appl. DNA Sci. http://adnas.com/signature-t/. Accessed 8 Jul 2018

40. Richero R, Ferrigno S (2016) A background analysis on transparency and traceability in the garment value chain. Directorate General for International Cooperation and Development, European Commission, Brussels.

41. Oxford University Press (2018) security | Definition of security in English by Oxford Dictionaries. In: Oxf. Dictionaries Engl. https:// en.oxforddictionaries.com/definition/security. Accessed 8 Jul 2018.

42. Li J, Lu C, Wang A et al (2016) Experimental investigation and mathematical modeling of laser marking two-dimensional barcodes on surfaces of aluminum alloy. J Manuf Process 21:141-152. https://doi.org/10.1016/j.jmapro.2015.12.007

43. Philip B, Jewell E, Greenwood P, Weirman C (2016) Material and process optimization screen printing carbon graphite pastes for mass production of heating elements. J Manuf Process 22:185191. https://doi.org/10.1016/j.jmapro.2016.03.001

44. Gennaro R (2006) Randomness in cryptography. IEEE Secur Priv 4:64-67. https://doi.org/10.1109/MSP.2006.49

45. Vedel-Smith NK, Lenau TA (2012) Casting traceability with direct part marking using reconfigurable pin-type tooling based on paraffin-graphite actuators. J Manuf Syst 31:113-120. https://doi.org/ 10.1016/j.jmsy.2011.12.001

46. Ranjan R, Khan AR, Parikh C et al (2016) Classification and identification of surface defects in friction stir welding: an image processing approach. J Manuf Process 22:237-253. https://doi.org/10. 1016/j.jmapro.2016.03.009

47. Katayama A, Nakamura T, Yamamuro M, Sonehara N (2004) New high-speed frame detection method: Side Trace Algorithm (STA) for I-appli on cellular phones to detect watermarks. In: Proceedings of the 3rd International Conference on Mobile and Ubiquitous Multimedia. ACM, New York, NY, USA, pp 109-116

48. Nakamura T, Katayama A, Yamamuro M, Sonehara N (2006) Fast watermark detection scheme from camera-captured images on mobile phones. Int J Pattern Recognit Artif Intell 20:543-564. https:// doi.org/10.1142/S0218001406004818

49. Sonka M, Hlavac V, Boyle R (1993) Image pre-processing. In: Image processing, analysis and machine vision. Springer, Boston, MA, pp 56-111

50. Otsu N (1979) A threshold selection method from gray-level histograms. IEEE Trans Syst Man Cybern 9:62-66

51. Soille P (1999) Morphological image analysis - principles and applications. Springer-Verlag, Berlin, pp 173-174

52. Duda RO, Hart PE (1972) Use of the Hough transformation to detect lines and curves in pictures. Commun ACM 15:11-15. https://doi.org/10.1145/361237.361242

53. Sonka M, Hlavac V, Boyle R (2014) Image processing, analysis, and machine vision. Cengage Learning.

54. Prewitt JMS, Mendelsohn ML (1966) The analysis of cell images. Ann N Y Acad Sci 128:1035-1053. https://doi.org/10.1111/j.17496632.1965.tb11715.x

55. Surový P, Dinis C, Marušák R, Ribeiro N de A (2014) Importance of automatic threshold for image segmentation for accurate measurement of fine roots of woody plants. Cent Eur For J 60: doi: https://doi.org/10.1515/forj-2015-0007

56. Aurenhammer F (1991) Voronoi diagrams - a survey of a fundamental geometric data structure. ACM Comput Surv 23:345-405. https://doi.org/10.1145/116873.116880

57. Zadeh LA (1975) Fuzzy logic and approximate reasoning. Synthese 30:407-428. https://doi.org/10.1007/BF00485052 